\title{
Biliary papillomatosis
}

Hong Kong Med J 2014;20:168.e3

YQ Hsu *

DOI: 10.12809/hkmj133976

A 65-year-old man presented with cholangitis which was treated effectively with antibiotics in October 2012. Abdominal computed tomography revealed gallstones inside the gallbladder; the intrahepatic duct (IHD) and common bile duct (CBD) were dilated but no stones were detected therein. However, a mural lesion was suspected inside the upper CBD. Subsequently, endoscopic retrograde cholangiopancreatography (ERCP) was performed and revealed dilated IHD and CBD, and multiple filling defects throughout the entire dilated CBD (Fig a). The right and left hepatic ducts were dilated but no filling defects were seen. After biliary sphincterotomy, and sweeping of the CBD with a balloon catheter, abundant gelatinous material mixed with tissue and three small pigmented stones were extracted through the papilla. Repeated sweeping of the CBD and imaging showed an extensive frondy mass attached to the CBD wall floating inside the lumen

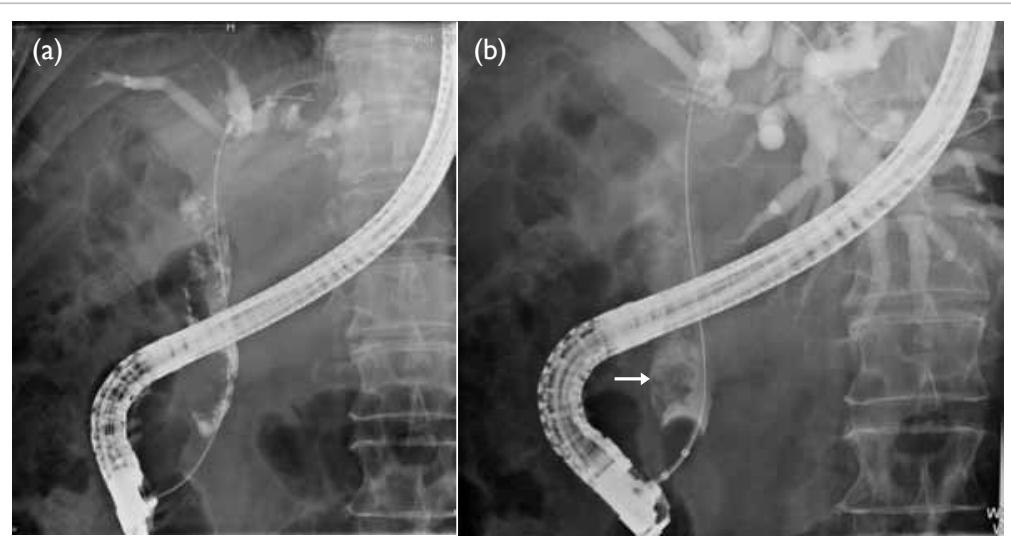

FIG. Endoscopic retrograde cholangiopancreatogram view showing (a) multiple irregular filling defects inside the common bile duct (CBD), and (b) an extensive frondy mass (arrow) attached to the CBD wall floating inside the lumen
(Fig b). Some tissues were extracted for histological examination which revealed dysplastic cells. The ERCP findings were highly suggestive of the diagnosis of intraductal papillary mucinous neoplasm. Because of its malignant potential, resection of extrahepatic bile duct was performed. Pathological examination showed the CBD was extensively involved by highgrade dysplastic glands forming papillomatosis; no invasive malignancy was seen. The patient recovered uneventfully after the operation.

Biliary papillomatosis ${ }^{1}$ is a rare disorder characterised by multiple papillary adenomas in the biliary tree. It affects mainly middle-aged, or elderly persons and commonly presents with obstructive jaundice and cholangitis. The papillomatosis varies in extent and distribution within the intrahepatic and/or extrahepatic biliary tree. The papillomas can be classified into mucin or non-mucin secreting, and are premalignant with definite malignant potential. The pathogenesis of this condition is unknown, although it has been suggested that the malignant transformation follows the pathway of adenoma to carcinoma sequence, similar to colonic polyps adenoma. The definitive treatment is surgical resection.

YQ Hsu *, FRCP, FHKAM (Medicine)

Department of Medicine, St. Teresa's Hospital, 327 Prince Edward Road West, Kowloon, Hong Kong

* Corresponding author: hsuyauque@yahoo.com

\section{Reference}

1. Lee SS, Kim MH, Lee SK, et al. Clinicopathologic review of 58 patients with biliary papillomatosis. Cancer 2004;100:783-93. 